DOI 10. 18307/2018. 0308

(C) 2018 by Journal of Lake Sciences

\title{
三峡库区水体和底泥中多环芳烃和邻苯二甲酸酯类分布和来源”
}

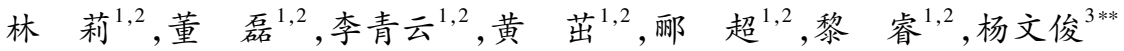 \\ (1: 长江科学院流域水环境研究所,武汉 430010) \\ (2: 长江科学院流域水资源与生态环境科学湖北省重点实验室,武汉 430010) \\ (3: 长江科学院院长办公室,武汉 430010)
}

\begin{abstract}
摘 要: 通过对 2016 年三峡库区干支流 18 个采样点水体和底泥中 16 种多环芳烃 (PAHs) 和 6 种邻苯二甲酸酯类 (PAEs) 污染物浓度的时空分布特征和来源进行分析, 得出如下结论:三峡库区 2016 年水体和底泥中 $\Sigma$ PAHs 分别为 $3.9 \sim$ $107.6 \mathrm{ng} / \mathrm{L}$ (均值为 $39.9 \mathrm{ng} / \mathrm{L}$ ) 和 $267.9 \sim 1018.1 \mathrm{ng} / \mathrm{g}$ (均值为 $490.9 \mathrm{ng} / \mathrm{g}$ ), $\Sigma \mathrm{PAEs}$ 分别为 $122.4 \sim 2884.7 \mathrm{ng} / \mathrm{L}$ (均值为 $848.1 \mathrm{ng} / \mathrm{L}$ ) 和 $192.9 \sim 3473.4 \mathrm{ng} / \mathrm{g}$ (均值为 $1253.35 \mathrm{ng} / \mathrm{g}$ ). 水库水体和底泥中 PAHs 和 PAEs 均表现出显著的时空分布特 征. 干支流水体 $\Sigma \mathrm{PAHs}$ 平均浓度均为放水期 ( 6 月 ) 高于蓄水期 (12 月), 干流底泥 $\Sigma \mathrm{PAHs}$ 平均含量在蓄水期高于放水 期. 干流水体中 $\Sigma$ PAEs 平均浓度在蓄水期显著高于放水期, 底泥中 $\Sigma$ PAEs 平均含量为放水期高于蓄水期. 库区水体中的 PAHs 以 2 3 环和 4 环为主, 底泥中以 4 环和 5 6 环为主. 水体和底泥中 PAEs 均以邻苯二甲酸 (2-乙基已基) 酯和邻苯 二甲酸二正丁酯为主. 库区水体中 PAHs 的主要来源为焦化或煤焦油挥发、石油源及燃料的中低温燃烧; 底泥中 PAHs 主 要来源为煤和生物质燃烧以及石油. 水体和底泥中的 PAEs 主要来源于塑料和重化工工业以及生活垃圾.
\end{abstract}

关键词: 三峡水库;多环芳烃 $(\mathrm{PAHs})$; 邻苯二甲酸酯类 (PAEs) ; 分布; 污染来源

\section{Distribution and sources of polycyclic aromatic hydrocarbons and phthalic acid esters in water and surface sediment from the Three Gorges Reservoir}

\author{
LIN Li ${ }^{1,2}$, DONG Lei ${ }^{1,2}$, LI Qingyun ${ }^{1,2}$, HUANG Zhuo ${ }^{1,2}$, LI Chao ${ }^{1,2}$, LI Rui ${ }^{1,2}$ \& YANG Wenjun ${ }^{3 * *}$ \\ (1: Basin Water Environmental Research Department, Changjiang River Scientific Research Institute, Wuhan 430010, P.R. \\ China) \\ (2: Hubei Provincial Key Lab of Basin Water Resource and Eco-Environmental Science, Changjiang River Scientific Research \\ Institute, Wuhan 430010, P.R. China) \\ (3: Administration Office, Changjiang River Scientific Research Institute, Wuhan 430010, P.R.China)
}

\begin{abstract}
Distribution and potential sources of 16 polycyclic aromatic hydrocarbons (PAHs) and 6 phthalic acid esters (PAEs) at 18 sampling sites during water drawdown and impoundment period were investigated in surface water and sediment from the Three Gorges Reservoir ( TGR) in 2016. In surface water, the $\Sigma$ PAHs concentrations in the TGR were 3.9-107.6 ng/L ( mean value was $39.9 \mathrm{ng} / \mathrm{L}$ ) and 267.9-1018.1 ng/g (mean value was $490.9 \mathrm{ng} / \mathrm{g}$ ) in water and sediment, respectively. The $\Sigma$ PAEs concentrations were $122.4-2884.7 \mathrm{ng} / \mathrm{L}$ ( mean value was $848.1 \mathrm{ng} / \mathrm{L}$ ) and $192.9-3473.4 \mathrm{ng} / \mathrm{g}$ ( mean value was $1253.35 \mathrm{ng} / \mathrm{g}$ ) in water and sediment, respectively. PAHs and PAEs show significant spatial variations in the TGR. The mean $\Sigma$ PAHs concentration in water during water drawdown period (June) was higher than that during water impoundment period (December) both in mainstream and tributaries, and the mean $\Sigma$ PAHs concentration in sediment during water impoundment period was higher than that during water drawdown period in mainstream. The mean $\Sigma$ PAEs concentration in sediment during water impoundment period was higher than that during water drawdown period in mainstream. Whereas, the mean $\Sigma$ PAHs concentration in sediment during water drawdown period was higher than that during water impoundment period in mainstream. PAH monomers with ( $2+3$ )-ring and 4-ring were
\end{abstract}

* 水利部公益性行业专项经费项目 (201501042)、中国科协青年人才托举工程项目 (2015QNRC001) 和中央级公益性 科研院所基本科研业务费项目 (CKSF2017062/SH，CKSF2015014/SH) 联合资助. 2017-06-27 收稿; 2017-08-22 收修改稿. 林莉(1983 ), 女, 博士, 高级工程师; E-mail:linli1229@ hotmail.com.

** 通信作者;E-mail:yangwj@ mail.crsri.cn. 
dominant in water, and with 4-ring and (5+6)-ring were dominant in sediment. Di-n-butyl phthalate and di-2-ethylhexyl phthalate were the dominant PAE pollutants in the TGR. The main source of PAHs in water is a mixture of creosote or coal tar volatilizations, petroleum sources and low temperature combustions of fuels. Whereas, the main source of PAHs in sediments is a mixture of coal and biomass combustions and petroleum sources, and petroleum combustions. The main sources of PAEs in the TGR was plastics and heavy chemical industries, and domestic wastes.

Keywords: Three Georges Reservoir; polycyclic aromatic hydrocarbons (PAHs); phthalic acid esters (PAEs); distribution; source of pollution

多环芳烃 $(\mathrm{PAHs})$ 和邻苯二甲酸酯类 $(\mathrm{PAEs})$ 是环境中广泛存在的持久性有毒污染物 ${ }^{[1-6]}$. PAHs 具有毒 性和致癌作用, 主要来源于含碳物质的不完全燃烧 ${ }^{[7]}$. PAEs 作为增塑剂被广泛使用 ${ }^{[8]}$, 属于内分泌干扰 物 ${ }^{[3,9]}$. 我国《地表水环境质量标准》(GB 3838-2002) 对地表水中 PAHs 和 PAEs 浓度均有限制要求 ${ }^{[10]}$.

三峡工程是世界上最大的水利枢纽工程, 在防洪、发电、航运和供水方面发挥了显著的效益 ${ }^{[11]}$.三峡水 库的水质对于人类和生态系统健康具有重要意义. 从天然状态至 $175 \mathrm{~m}$ 蓄水, 三峡水库水文情势发生了巨 大变化, 水流减缓显著降低了水中污染物的扩散和自净能力, 污染物的沉降和运移规律也相应改变 ${ }^{[12]}$. 三 峡水库的水质问题日益引起广泛关注.

$\mathrm{PAHs}$ 被认为是三峡库区首要的有毒污染物 ${ }^{[12-14]}$. 目前关于三峡库区水体和底泥中 PAHs 的浓度和来 源虽然已有研究 ${ }^{[7,15-16]}$, 但长期跟踪监测和分析对于库区水质管理十分必要. 据报道 PAEs 是三峡工程 175 $\mathrm{m}$ 蓄水前库区水体中的主要污染物之一 ${ }^{[17]}$. 2005 年, 邻苯二甲酸二正丁酯 (DBP) 和邻苯二甲酸 (2-乙基已 基) 酯( DEHP) 浓度分别为 $0.83 \sim 2.21$ 和 $0.66 \sim 3.60 \mu \mathrm{g} / \mathrm{L}^{[17]}$. 关于三峡工程 $175 \mathrm{~m}$ 蓄水后库区水体和底泥 中 PAEs 浓度和污染来源的报道极少. 开展三峡工程蓄水后库区主要持久性有机污染物 PAHs 和 PAEs 在水 体和底泥中的含量、分布与来源研究, 对于三峡库区生态环境安全具有重要意义.

本文系统研究了三峡水库水体和底泥中 PAHs 和 PAEs 的浓度、分布和主要来源. 采集了 2016 年三峡 水库放水期 (6月) 和蓄水期 (12 月) 干支流 18 个采样点的水样和底泥样品, 分析了不同水期下污染物的时 空分布和来源特征. 调查的污染物包括美国环境保护局 (EPA) 优先污染物名单中所列的 16 种 PAHs 和 6 种 PAEs. 本研究结果可为三峡库区未来开展水质监测和污染控制提供理论依据.

\section{1 材料和方法}

\section{1 化学品和试剂}

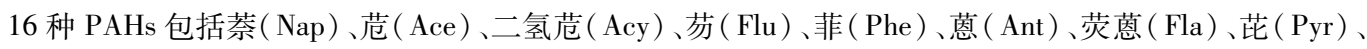
苯并葱 $(\mathrm{BaA})$ 、屈 $(\mathrm{Chr})$ 、苯并 $[\mathrm{b}]$ 苂葱 $(\mathrm{BbF})$ 、苯并 $[\mathrm{k}]$ 苂葱 $(\mathrm{BkF})$ 、苯并 $[\mathrm{a}]$ 萠 $(\mathrm{BaP}) 、$ 、狮并萠 $(\mathrm{InP})$ 、二苯并 $[\mathrm{a}, \mathrm{h}]$ 葱 $($ DahA $)$ 和苯并 $[\mathrm{g}, \mathrm{h}, \mathrm{i}]$ 萠 (BghiP). 6 种 PAEs 包括邻苯二甲酸二甲酯 (DMP)、邻苯二甲酸二乙酯 (DEP)、邻苯二甲酸二正丁酯 (DBP)、邻苯二甲酸丁基芐酯 (BBP)、邻苯二甲酸 (2-乙基已基) 酯 (DEHP) 和 邻苯二甲酸二正辛酯 (DNOP). PAHs 混合标准储备液 ( Lot 214101426, 99\%) 和 PAEs 混合标准储备液 ( Lot 214031130, 99\% ) 均由 AccuStandard 公司提供. 二氯甲烷、丙酮和乙酸乙酯均为色谱纯, 由美国 Fisher Chemical 公司提供. 正已烷为色谱纯,由美国 TEDIA 公司提供.

\section{2 样品采集}

本研究共设置三峡库区干流 14 个采样点 (M1 M14) 和支流 4 个采样点 (T1 T4) (图 1). 水样和底泥 样品分别采集于 2016 年 6 月 (放水期) 和 12 月 (蓄水期). 4 条支流包括乌江、渠溪河、梅溪河和青干河. 采 用 $4 \mathrm{~L}$ 不锈钢小桶采集 $0 \sim 50 \mathrm{~cm}$ 表层水体,采集后过 $0.45 \mu \mathrm{m}$ 滤膜并保存于 $4 \mathrm{~L}$ 棕色瓶中. 采用抓斗式采泥 器采集表层底泥, 采集后保存于封口袋中. 所有的水体和底泥样品尽快运回实验室, 并于 $4^{\circ} \mathrm{C}$ 保存待分析.

\section{3 预处理和分析方法}

采用 C18 固相萃取膜 (ENVI-18 DSK, $47 \mathrm{~mm}$ 直径, Sigma-Aldrich, USA) 萃取水体样品中的 PAHs 和 $\mathrm{PAEs}^{[18-19]}$. 萃取后固相萃取膜采用 $10 \mathrm{ml}$ 乙酸乙酯洗脱 1 次,加 $10 \mathrm{ml}$ 乙酸乙酯重复洗脱 1 次,再采用 $10 \mathrm{ml}$ $(1+1)$ 二氯甲烷-乙酸乙酯洗脱 1 次, 以上洗脱步骤重复 2 次. 将所有洗脱液收集后,除水并浓缩至近干,用 正已烷定容至 $1.0 \mathrm{ml}$,上机分析. 


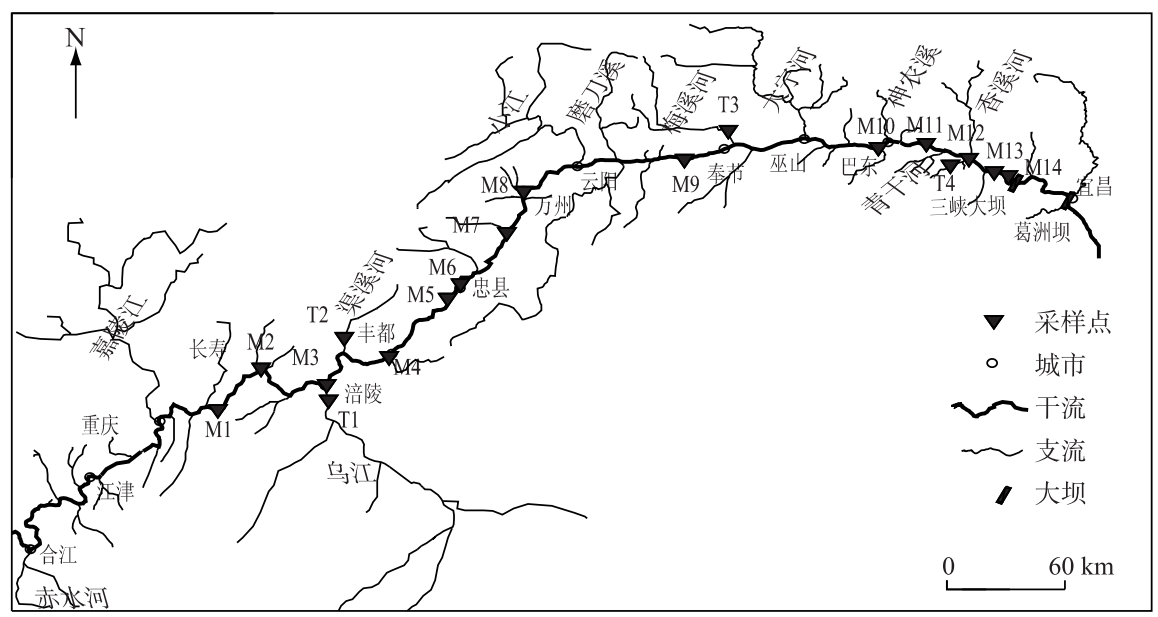

图 1 三峡库区采样点分布

Fig.1 Distribution of sampling sites in the Three Georges Reservoir

底泥样品经真空冷冻干燥、研磨、过篮, 取约 $2 \mathrm{~g}$ ( 精确到 $0.0001 \mathrm{~g}$ ) 加人 $25 \mathrm{ml}(1+1)$ 己烷-丙酮混合溶 剂, 采用微波萃取. 微波萃取条件为: 自室温 $\left(30^{\circ} \mathrm{C}\right)$ 以 $10^{\circ} \mathrm{C} / \mathrm{min}$ 的速度升温至 $120^{\circ} \mathrm{C}$, 保持 $20 \mathrm{~min}$. 硅胶一氧 化铝复合柱采用湿法填柱, 自下而上依次填人 $0.5 \mathrm{~cm}$ 无水硫酸钠、 $3 \mathrm{~cm}$ 中性氧化铝、 $3 \mathrm{~cm}$ 中性硅胶和 $1 \mathrm{~cm}$ 无水硫酸钠, 净化柱用 $10 \mathrm{ml}$ 正己烷 $10 \mathrm{ml}(7+3)$ 正已烷与二氯甲烷混合溶剂预冲洗. 将萃取液通过硅胶一 氧化铝复合柱, 然后采用 $20 \mathrm{ml}$ 正己烷、20 ml(7+3) 正已烷与二氯甲烷混合溶剂淋洗柱子. 将所有洗脱液收 集后,出水并浓缩至近干,用正已烷定容至 $1.0 \mathrm{ml}$, 上机分析.

16 种 PAHs 和 6 种 PAEs 采用 GC-MS(7890B/5977A, Agilent, USA) 检测. 采用 DB-5MS 色谱柱, 质谱条 件为 $\mathrm{EI}$ 模式, 离子源温度为 $250^{\circ} \mathrm{C}$; 扫描方式为 $\mathrm{SIM}$, 载气均为高纯氮气 ( $\left.\geqslant 99.999 \%\right)$, 不分流进样, 进样体 积 $1 \mu \mathrm{l}$. PAHs 检测色谱条件为: 进样口温度 $250^{\circ} \mathrm{C}$ 、传输线温度 $300^{\circ} \mathrm{C}$ 、流速 $1 \mathrm{ml} / \mathrm{min}$; 升温程序为: $80^{\circ} \mathrm{C}$ 保持 $2 \mathrm{~min}$, 以 $20^{\circ} \mathrm{C} / \mathrm{min}$ 升温至 $220^{\circ} \mathrm{C}$, 保持 $10 \mathrm{~min}$, 再以 $2^{\circ} \mathrm{C} / \mathrm{min}$ 升温至 $300^{\circ} \mathrm{C}$, 保持 $5 \mathrm{~min}$. PAEs 检测色谱条件 为: 进样口温度 $250^{\circ} \mathrm{C}$ 、传输线温度 $300^{\circ} \mathrm{C}$ 、流速 $1.2 \mathrm{ml} / \mathrm{min}$; 升温程序为: $70^{\circ} \mathrm{C}$ 保持 $2 \mathrm{~min}$, 以 $20^{\circ} \mathrm{C} / \mathrm{min}$ 升温至 $130^{\circ} \mathrm{C}$, 再以 $5^{\circ} \mathrm{C} / \mathrm{min}$ 升温至 $200^{\circ} \mathrm{C}, 15^{\circ} \mathrm{C} / \mathrm{min}$ 升温至 $300^{\circ} \mathrm{C}$, 保持 $5 \mathrm{~min}$.

\section{4 质量控制}

16 种 PAHs 和 6 种 PAEs 采用外标法进行质量控制. 为避免分析过程中引起的污染, 每分析 10 个样品 做一个样品空白. 样品上机分析测试时按 $10 \%$ 比例抽取样品开展平行试验. 所有样品均上机测试 3 次并取 平均值. 所有标准曲线相关系数均大于 0.994. 底泥样品中 PAHs 和 PAEs 含量均以干重法计算 $(\mathrm{ng} / \mathrm{g}(\mathrm{dw}))$. 水体样品中 16 种 PAHs 的方法检出限为 $0.04 \sim 0.39 \mathrm{ng} / \mathrm{L}$, 底泥样品中 16 种 PAHs 的方法检出限为 $0.08 \sim$ $0.78 \mathrm{ng} / \mathrm{g}$. 水样中 6 种 PAEs 的方法检出限为 $0.12 \sim 0.92 \mathrm{ng} / \mathrm{L}$, 底泥样品中 6 种 PAEs 的方法检出限为 $0.25 \sim$ $1.85 \mathrm{ng} / \mathrm{g}$. 水体和底泥样品中 16 种 PAHs 的加标回收率分别为 $80.34 \% \sim 112.06 \%$ 和 $59.87 \% \sim 80.75 \%, 6$ 种 PAEs 的加标回收率分别为 $86.90 \% \sim 110.12 \%$ 和 $63.93 \% \sim 75.54 \%$.

\section{5 数据分析}

采用主成分分析法 (PCA) 研究三峡库区水体和底泥中 PAHs 和 PAEs 可能的来源. 采用 IBM SPSS Statistics 20 软件进行主成分分析.

\section{2 结果与讨论}

\section{1 水体和底泥中 PAHs 的分布及来源}

2.1.1 PAHs 的分布从表 1 可以看出, 2016 年三峡库区水体中 $\Sigma \mathrm{PAHs}$ 浓度范围为 3.9 107.6 $\mathrm{ng} / \mathrm{L}$ ( 均值为 $39.9 \mathrm{ng} / \mathrm{L}$ ), 低于 2011 年 (范围为 $18 \sim 159 \mathrm{ng} / \mathrm{L}$, 均值为 $44.1 \mathrm{ng} / \mathrm{L}$ ) ${ }^{[12]}$, 以及 2012 年 (范围为 $131 \sim 228 \mathrm{ng} / \mathrm{L}$, 
均值为 $155 \mathrm{ng} / \mathrm{L})^{[14]}$. 水体中 $\Sigma \mathrm{PAHs}$ 平均浓度在支流高于干流, 且干流和支流均为放水期高于蓄水期. 2016 年三峡库区底泥中 $\sum$ PAHs 含量范围为 $267.9 \sim 1018.1 \mathrm{ng} / \mathrm{g}$ (均值为 $490.9 \mathrm{ng} / \mathrm{g}$ ), 远低于 2005 年长江武汉段 底泥中 $\sum$ PAHs 含量均值 $(1334.5 \mathrm{ng} / \mathrm{g})^{[20]}$, 高于 2010-2011 年长江口底泥中 $\sum$ PAHs 含量 $(128.5 \sim 307.8 \mathrm{ng} /$ $\mathrm{g})^{[7]}$. 干流和支流底泥中 $\Sigma \mathrm{PAHs}$ 平均含量差异不大. 干流中底泥 $\Sigma \mathrm{PAHs}$ 平均含量在蓄水期高于放水期, 可 能是由于蓄水期水体中 PAHs 的沉淀作用所致 ${ }^{[12]}$.

表 1 三峡库区水体和底泥中的 $\Sigma P A H s$ 分布

Tab.1 $\mathrm{\Sigma PAHs}$ in water and sediment from the TGR

\begin{tabular}{|c|c|c|c|c|c|}
\hline & & \multicolumn{2}{|c|}{ 水体 $\Sigma \mathrm{PAHs} /(\mathrm{ng} / \mathrm{L})$} & \multicolumn{2}{|c|}{ 底泥 $\Sigma$ PAHs/ (ng/g) } \\
\hline & & 6 月 & 12 月 & 6 月 & 12 月 \\
\hline \multirow{5}{*}{$\begin{array}{c}\text { 所有样点 } \\
\text { ( M1 M14, } \\
\text { T1 T4) }\end{array}$} & 最小值 & 5.2 & 3.9 & 267.9 & 287.1 \\
\hline & 最大值 & 107.6 & 52.1 & 1018.1 & 991.0 \\
\hline & 平均值 & 63.5 & 16.2 & 457.4 & 524.5 \\
\hline & 中值 & 64.3 & 14.6 & 397.3 & 517.6 \\
\hline & 标准偏差 & 25.3 & 10.3 & 173.6 & 162.5 \\
\hline \multirow{5}{*}{$\begin{array}{c}\text { 干流 } \\
(\mathrm{M} 1 \sim \mathrm{M} 14)\end{array}$} & 最小值 & 5.2 & 4.3 & 267.9 & 287.1 \\
\hline & 最大值 & 88.4 & 52.1 & 1018.1 & 991.0 \\
\hline & 平均值 & 58.1 & 16.5 & 457.5 & 553.0 \\
\hline & 中值 & 61.3 & 14.3 & 397.3 & 552.9 \\
\hline & 标准偏差 & 23.0 & 11.1 & 189.2 & 169.7 \\
\hline \multirow{5}{*}{$\begin{array}{c}\text { 支流 } \\
(\mathrm{T} 1 \sim \mathrm{T} 4)\end{array}$} & 最小值 & 43.1 & 3.9 & 348.5 & 314.6 \\
\hline & 最大值 & 107.6 & 21.9 & 601.7 & 510.8 \\
\hline & 平均值 & 82.3 & 15.5 & 456.8 & 424.8 \\
\hline & 中值 & 89.3 & 18.1 & 438.5 & 436.9 \\
\hline & 标准偏差 & 24.0 & 6.9 & 102.0 & 72.5 \\
\hline
\end{tabular}

16 种 PAHs 可根据含有的苯环数量被划分为 $2 \sim 3$ 环、4 环和 $5 \sim 6$ 环, 分别代表低分子、中分子和高分子 $\mathrm{PAHs}^{[21]}$. 放水期水体中主要的 PAH 单体是 $2 \sim 3$ 环, 蓄水期为 $2 \sim 3$ 环和 4 环. 不论蓄水期还是放水期, Nap 均 为水体中浓度最高的 PAH. 长江干流武汉段水体中主要的 PAH 单体为 $2 \sim 3$ 环和 4 环 ${ }^{[20]}$, 与三峡库区较相似. 各采样断面检出的 $\mathrm{BaP}$ 含量范围为 $0 \sim 0.64 \mathrm{ng} / \mathrm{L}$, 未超过我国《地表水环境质量标准》限值 $2.8 \mathrm{ng} / \mathrm{L}^{[10]}$. 底泥 中, 放水期比蓄水期 PAH 单体浓度变化幅度大, PAH 单体除了 Phe 含量较高外, 其余均以 4 环和 $5 \sim 6$ 环为主 (图 2). 综合分析, 三峡库区水体中 PAH 以 2 3 环和 4 环为主, 底泥中以 4 环和 5 6 环为主, 可能是因为环数 较小的 PAHs 溶解度较大, 不容易沉积到底部;而环数大的 PAHs 疏水性强, 更容易被底泥吸附.

2.1.2 PAHs 的来源辨识水体和底泥中 PAHs 的来源对于水质管理十分重要. 主成分分析常被用于分析 $\mathrm{PAHs}$ 的来源 ${ }^{[7,22]}$. 三峡库区水体中的 PAHs 共提取了 3 种主成分 $(\mathrm{PC})$, 主成分的提取以特征根大于 1 为标 准. 由于 6 月和 12 月水样中 PC3 的方差贡献率较低, 分别仅为 $6.10 \%$ 和 $9.52 \%$, 而 PC1 和 PC2 的累积方差 分别达到了 $87.41 \%$ 和 $82.88 \%$, 可以代表水体和底泥中 PAHs 的大多数信息 (图 3). 因此图 3 中未展示 PC3 的结果, 仅显示 PC1 和 PC2 的结果. 底泥样品在 6 和 12 月均只提取出 1 个主成分, PC1 的方差贡献率分别 达到 $88.90 \%$ 和 $86.28 \%$.

水体中 Nap 在两个水期均为 PC1 的主要贡献者. Flu 和 Ant 分别是放水期和蓄水期样品中 PC2 的主要 贡献者 (图 3). 由于 Nap 是焦化或煤焦油挥发的特征标志物 ${ }^{[22]}$, 通常代表大气传输 ${ }^{[23]}$. Nap、Flu 和 Ant 均为 $2 \sim 3$ 环的 PAHs, 通常来自石油源及燃料的中低温燃烧 ${ }^{[23]}$. 因此,水体中 PAHs 的主要来源是焦化或煤焦油 挥发、石油源及燃料的中低温燃烧. 6 月底泥中 Phe 对 PC1 的贡献率最高, 12 月以 Fla 贡献率最高. Phe 与煤 和生物质燃烧排放有关, 同时与原油和石油的泄漏有关 ${ }^{[7]}$, 这表明 6 月底泥中 PC1 主要贡献为煤和生物质 燃烧以及石油. Fla 主要来源于工业燃煤和民用燃煤 ${ }^{[23]}$. 因此, 三峡库区底泥中 PAHs 主要的来源为煤和生 物质然烧以及石油. 

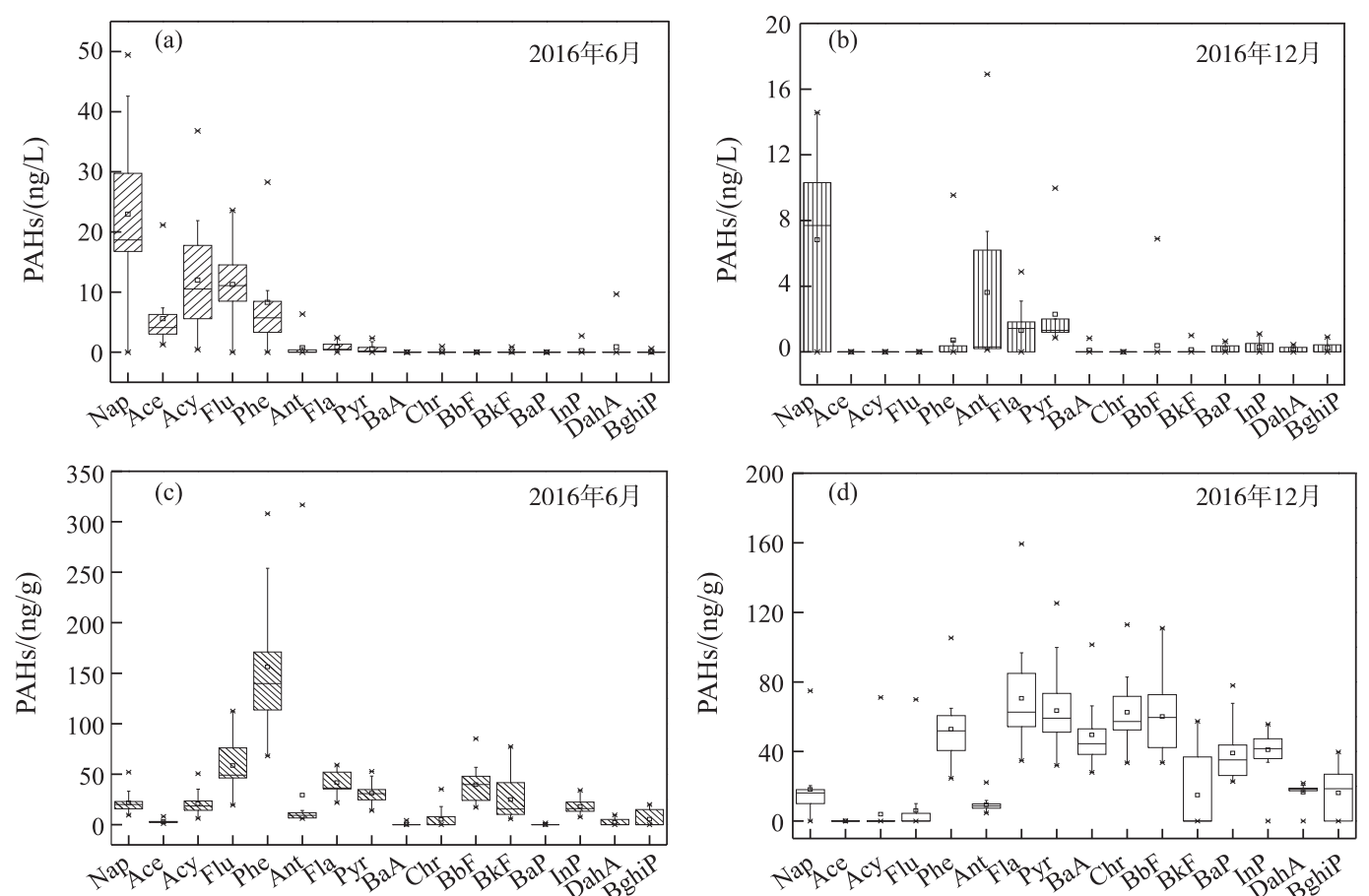

图 22016 年 6 月和 12 月三峡库区水体 $(\mathrm{a}$ 和 $\mathrm{b}$ ) 和底泥 $(\mathrm{c}$ 和 $\mathrm{d}$ ) 中 16 种 PAH 单体分布

Fig. 2 Distribution of 16 PAH monomers in water ( a, b) and sediment ( c, d) of the TGR in June and December of 2016
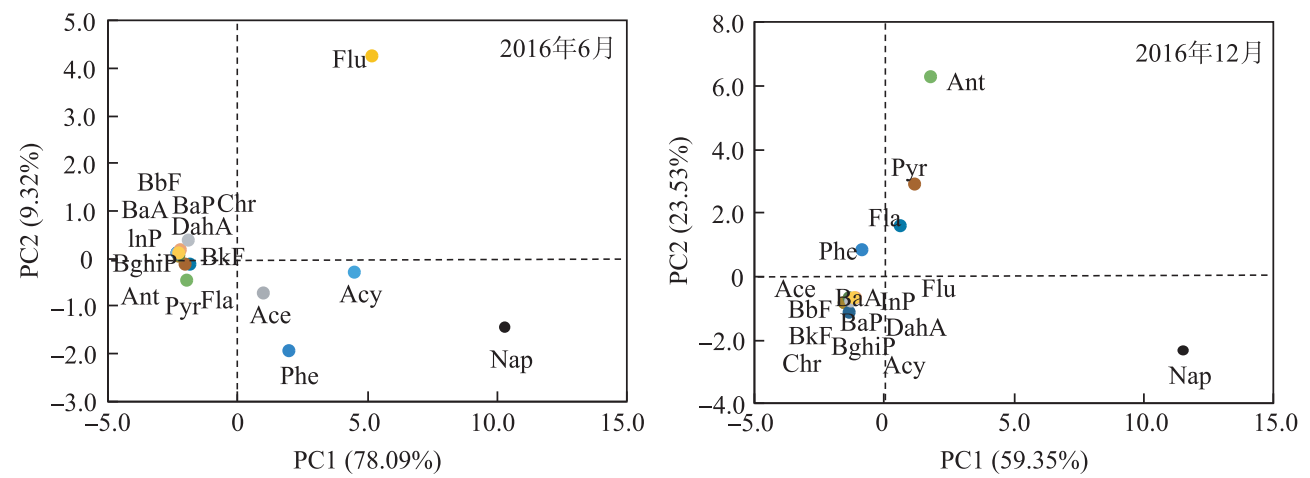

图 32016 年 6 月和 12 月三峡库区水体中 16 种 PAHs 的主成分分析

Fig.3 Rotated principal components of 16 PAHs in water of the TGR in June and December of 2016

\section{2 水体和底泥中 PAEs 的分布及来源}

2.2.1 PAEs 的分布 三峡库区 18 个采样点中均检测到 PAEs, 说明 PAEs 在三峡库区广泛存在. 水体中 $\Sigma P A E s$ 浓度范围为 $122.4 \sim 2884.7 \mathrm{ng} / \mathrm{L}$ ( 均值为 $848.1 \mathrm{ng} / \mathrm{L}$ ), 低于长江武汉段 (范围为 $34 \sim 91220 \mathrm{ng} / \mathrm{L}$, 均值 为 $23613 \mathrm{ng} / \mathrm{L}$ ) ${ }^{[24]}$ 以及长江口 (范围为 $61 \sim 28550 \mathrm{ng} / \mathrm{L}$, 均值为 $\left.4536 \mathrm{ng} / \mathrm{L}\right)^{[22]}$. 干流与支流水体中 $\Sigma \mathrm{PAEs}$ 平 均浓度差异不大. 干流水体中 $\Sigma \mathrm{PAEs}$ 的平均浓度在蓄水期显著高于放水期, 可能是蓄水期干流水体流速 慢、自净能力弱造成的 ${ }^{[24]}$. 支流水体中 $\Sigma$ PAEs 浓度在蓄水期和放水期无显著差异, 但放水期水体 $\Sigma$ PAEs 浓 度变化幅度大于蓄水期, 可能与外源污染物的汇人有关. 三峡库区底泥中 $\Sigma \mathrm{PAE}$ 浓度范围在放水期和蓄水 期份别为 $436.9 \sim 3127.7 \mathrm{ng} / \mathrm{g}$ (均值为 $1800.4 \mathrm{ng} / \mathrm{g}$ ) 和 $192.9 \sim 3473.4 \mathrm{ng} / \mathrm{g}$ (均值为 $706.3 \mathrm{ng} / \mathrm{g}$ ), 显著低于长 
江武汉段 $(76300 \sim 450000 \mathrm{ng} / \mathrm{g})^{[24]}$. 同时, 各 PAE 单体浓度也显著低于广州城市某湖泊底泥中各 PAHE 单 体浓度 ${ }^{[25]}$. 底泥中 $\Sigma$ PAEs 平均浓度在干流高于支流,放水期显著高于蓄水期 (表 2).

表 2 三峡库区水体和底泥中 $\Sigma$ PAEs 分布

Tab.2 Distribution of $\Sigma$ PAEs in water and sediment of the TGR

\begin{tabular}{|c|c|c|c|c|c|}
\hline & & \multicolumn{2}{|c|}{ 水体 $\Sigma$ PAEs $/(\mathrm{ng} / \mathrm{L})$} & \multicolumn{2}{|c|}{ 底泥 $\Sigma P A E s /(n g / g)$} \\
\hline & & 6 月 & 12 月 & 6 月 & 12 月 \\
\hline \multirow{5}{*}{$\begin{array}{c}\text { 所有样点 } \\
\text { ( M1 M14, } \\
\text { T1 T4) }\end{array}$} & 最小值 & 122.4 & 635.1 & 436.9 & 192.9 \\
\hline & 最大值 & 1577.0 & 2884.7 & 3127.7 & 3473.4 \\
\hline & 平均值 & 740.7 & 955.6 & 1800.4 & 706.3 \\
\hline & 中值 & 709.8 & 807.0 & 2022.1 & 477.7 \\
\hline & 标准偏差 & 345.5 & 492.7 & 822.7 & 716.6 \\
\hline \multirow{5}{*}{$\begin{array}{c}\text { 干流 } \\
(\mathrm{M} 1 \sim \mathrm{M} 14)\end{array}$} & 最小值 & 122.4 & 635.1 & 436.9 & 192.9 \\
\hline & 最大值 & 1246.9 & 2884.7 & 2959.8 & 3473.4 \\
\hline & 平均值 & 710.3 & 987.9 & 1918.4 & 742.7 \\
\hline & 中值 & 709.8 & 807.0 & 2061.6 & 461.3 \\
\hline & 标准偏差 & 294.5 & 552.1 & 707.3 & 801.2 \\
\hline \multirow{5}{*}{$\begin{array}{c}\text { 支流 } \\
(\mathrm{T} 1 \sim \mathrm{T} 4)\end{array}$} & 最小值 & 359.6 & 753.6 & 641.6 & 310.3 \\
\hline & 最大值 & 1577.0 & 983.8 & 3127.7 & 859.7 \\
\hline & 平均值 & 846.9 & 842.8 & 1298.8 & 579.0 \\
\hline & 中值 & 725.4 & 816.9 & 713.0 & 573.0 \\
\hline & 标准偏差 & 468.1 & 95.3 & 1056.8 & 208.5 \\
\hline
\end{tabular}

三峡库区水体中 DEHP 和 DBP 是主要污染物, 浓度范围分别为 $41.0 \sim 2425.6$ 和 $75.6 \sim 722.5 \mathrm{ng} / \mathrm{L}$. 所有 采样点水体中 DEHP 和 DBP 浓度均低于国家《地表水环境质量标准》的限值 $(8000 \text { 和 } 3000 \mathrm{ng} / \mathrm{L})^{[10]}$. 底泥 中主要污染物仍然为 DEHP 和 DBP, 含量分别为 $201.8 \sim 3278.46$ 和 $35.6 \sim 1796.3 \mathrm{ng} / \mathrm{g}$. 不论是水体还是底 泥, DEHP 均为浓度最高的 PAE 类污染物 (图 4).
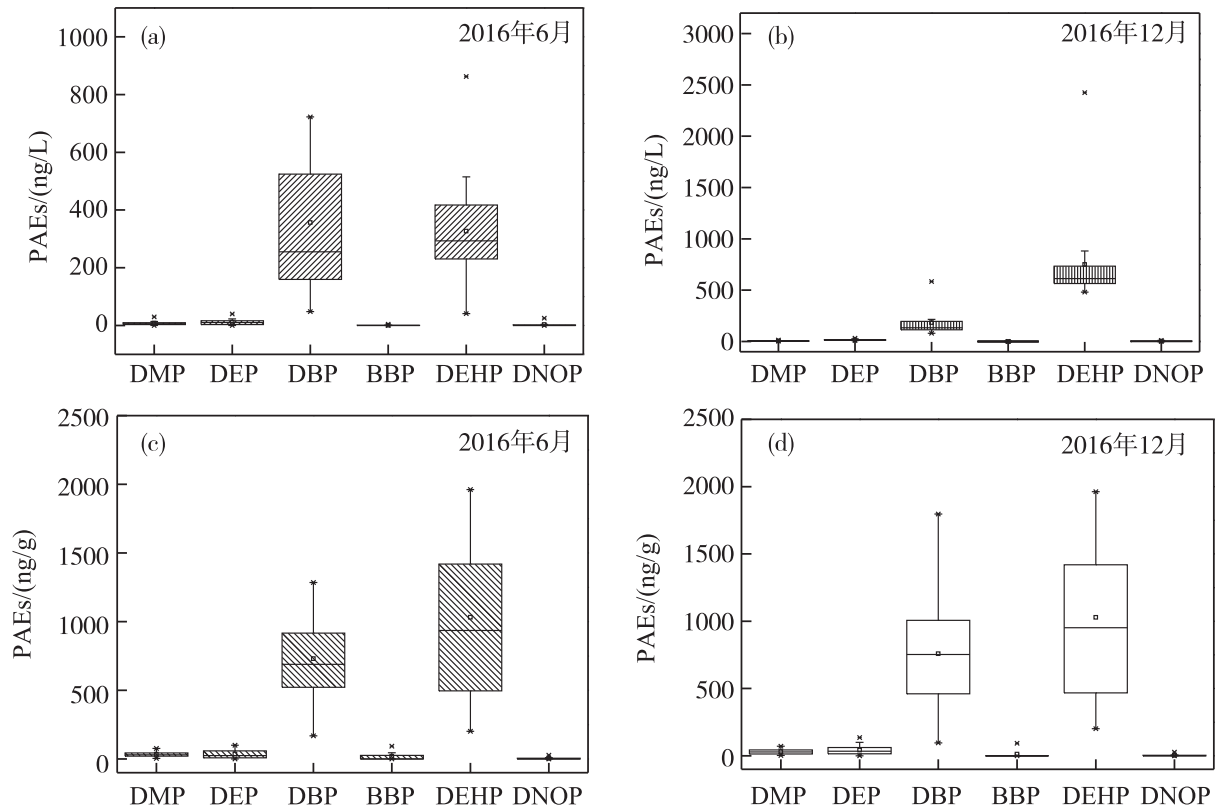

图 42016 年 6 月和 12 月三峡库区水体 ( $\mathrm{a}$ 和 $\mathrm{b}$ ) 和底泥 $(\mathrm{c}$ 和 $\mathrm{d}$ ) 中 6 种 PAE 单体分布

Fig.4 Distribution of 6 PAE monomers in water $(\mathrm{a}, \mathrm{b})$ and sediment $(\mathrm{c}, \mathrm{d})$ of the TGR in June and December of 2016 
2.2.2 PAEs 的来源 采用主成分分析法来分析三峡库区水体和底泥中 PAEs 的来源, 结果如图 5 所示. 对于 6 月水体和底泥样品, 均提取出 2 个主成分, 每个主成分的特征根均大于 1.6 月水体样品前两个主成分的方 差贡献率分别为 $92.60 \%$ 和 $7.28 \%$, 底泥样品分别为 $89.52 \%$ 和 $10.33 \%$. 对于 12 月的水体和底泥样品, 均提取 出 1 个主成分,PC1 的方差贡献率分别达到 $97.63 \%$ 和 $96.94 \%$.

6 月水体样品 PC1 中 DBP 和 DEHP 载荷最高, PC2 中 DBP 载荷最高; 12 月水体样品 PC1 中 DEHP 载荷 最高. 6 月底泥 PC1 中 DEHP 和 DBP 载荷最高, PC2 中 DBP 载荷最高; 12 月底泥样品 PC1 中 DEHP 载荷最 高. 总体而言, 水体和底泥样品中, 载荷最高的均为 DEHP, 其次为 DBP (图 5). DEHP 主要来源于塑料和重 化工产业, 同时也是家庭垃圾滤出液中主要的 PAE 类污染物 ${ }^{[22]}$. DBP 被广泛应用于化妆品和个人护理品 中 $^{[25]}$, 是生活垃圾中的主要 PAE 类污染物 ${ }^{[26]}$. 可见, 三峡库区水体和底泥中 PAEs 的主要来源于塑料和重 化工工业以及生活垃圾.
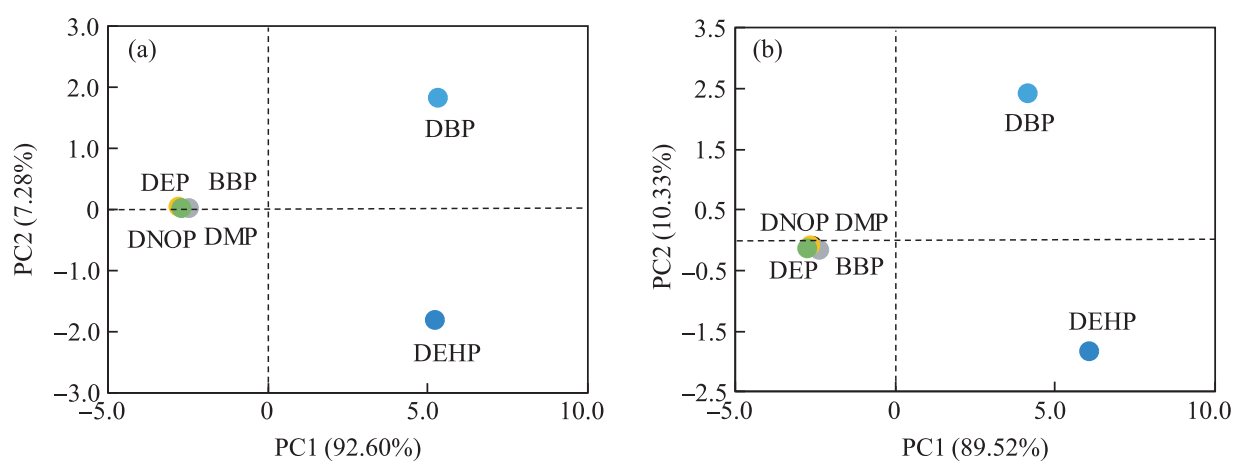

图 52016 年 6 月三峡库区水体 (a) 和底泥 (b) 中 6 种 PAE 的主成分分析

Fig.5 Rotated principal components of 6 PAEs in water (a) and sediment (b) of the TGR in June of 2016

\section{3 参考文献}

[ 1 ] Liu S, Xia X, Zhai Y et al. Black carbon (BC) in urban and surrounding rural soils of Beijing, China: Spatial distribution and relationship with polycyclic aromatic hydrocarbons (PAHs). Chemosphere, 2011, 82(2) : 223-228. DOI: 10. 1016/j.chemosphere.2010.10.017.

[ 2 ] Deyerling D, Wang J, Hu W et al. PAH distribution and mass fluxes in the Three Gorges Reservoir after impoundment of the Three Gorges Dam. Science of the Total Environment, 2014, 491/492: 123-130. DOI: 10.1016/j. scitotenv. 2014. 03.076 .

[ 3 ] Li B, Liu R, Gao H et al. Spatial distribution and ecological risk assessment of phthalic acid esters and phenols in surface sediment from urban rivers in Northeast China. Environmental Pollution, 2016, 219: 409. DOI: 10.1016/j.envpol.2016. 05.022 .

[ 4 ] Liu X, Shi J, Bo T et al. Occurrence and risk assessment of selected phthalates in drinking water from waterworks in China. Environ Sci Pollut Res, 2015, 22(14) : 10690-10698. DOI: 10.1007/s11356-015-4253-9.

[ 5 ] Net S, Sempéré R, Delmont A et al. Occurrence, fate, behavior and ecotoxicological state of phthalates in different environmental matrices. Environmental Science \& Technology, 2015, 49(7) : 4019. DOI: 10.1021/es505233b.

[ 6 ] Xu X, Tan Y, Yang G. Environmental impact assessments of the Three Gorges Project in China: Issues and interventions. Earth-Science Reviews, 2013, 124: 115-125. DOI: 10.1016/j.earscirev.2013.05.007.

[ 7 ] Wang Y, Shen C, Shen Z et al. Spatial variation and sources of polycyclic aromatic hydrocarbons (PAHs) in surface sediments from the Yangtze Estuary, China. Environmental Science Processes \& Impacts, 2015, 17 (7) : 1340-1347. DOI: 10. 1039/c5em00077g.

[ 8 ] Zhang L, Liu J, Liu H et al. The occurrence and ecological risk assessment of phthalate esters (PAEs) in urban aquatic environments of China. Ecotoxicology, 2015, 24(5) : 967-984. DOI: 10.1007/s10646-015-1446-4. 
[ 9 ] Xu Z, Zhang W, Lv L et al. A new approach to catalytic degradation of dimethyl phthlate by a macroporous $\mathrm{OH}$-type strongly basic anion exchange resin. Environmental Science \& Technology, 2010, 44 (8): 3130-3135. DOI: 10. $1021 /$ es100058j.

[10] GB 3838-2002. State Environmental Protection Administration of China: Environmental Quality Standard for Surface Water, 2002. [ GB 3838-2002. 中华人民共和国地表水环境质量标准, 2002.]

[11] Zhang K, Xiong X, Hu H et al. Occurrence and characteristics of microplastic pollution in Xiangxi Bay of Three Gorges Reservoir, China. Environmental Science \& Technology, 2017, 51(7) : 3794-3801. DOI: 10.1021/acs.est.7b00369.

[12] Wang J, Henkelmann B, Bi Y. Temporal variation and spatial distribution of PAH in water of Three Gorges Reservoir during the complete impoundment period. Environ. Sci Pollut Res, 2013, 20(10) : 7071-7079. DOI: 10.1007/s11356-0121427-6.

[13] Floehr T, Scholz-starke B, Xiao H et al. Yangtze Three Gorges Reservoir, China: A holistic assessment of organic pollution, mutagenic effects of sediments and genotoxic impacts on fish. J Environ Sci, 2015, 38: 63-82. DOI: 10.1016/j.jes. 2015.07.013.

[14] Zhu Y, Yang Y, Liu M et al. Concentration, Distribution, source, and risk assessment of pahs and heavy metals in surface water from the Three Gorges Reservoir, China. Hum Ecol Risk Assess A, 2015, 21 (6) : 1593-1607. DOI: 10. 1080/ 10807039.2014.962315.

[15] Tao. YQ, Yao SC, Xue B et al. Polycyclic aromatic hydrocarbons in surface sediments from drinking water sources of Taihu Lake, China: sources, partitioning and toxicological risk. J Environ Monit, 2010, 12(12): 2282-2289. DOI: 10. 1039/c0em00144a.

[16] Li SY, Tao YQ, Yao SC et al. Distribution, sources, and risks of polycyclic aromatic hydrocarbons in the surface sediments from 28 lakes in the middle and lower reaches of the Yangtze River region, China. Environ Sci Pollut R, 2016, 23 ( 5 ) : 4812-4825. DOI: $10.1007 / \mathrm{s} 11356-015-5705-\mathrm{y}$.

[17] Xu C, Shu WQ, Luo CH et al. Water environmental health risk assessment of PAHs and PAEs in the Three Gorges Reservoir. Res Environ Sci, 2007, 20(5) : 57-60. [许川, 舒为群, 罗财红等. 三峡库区水环境多环芳烃和邻苯二甲酸酯 类有机污染物健康风险评价, 环境科学研究, 2007, 20(5) : 57-60.]

[18] Limam I, Driss MR. Off-line solid-phase extraction procedure for the determination of polycyclic aromatic hydrocarbons from aqueous matrices. Int J Environ Sci Technol, 2013, 10(5) : 973-982. DOI: 10.1007/s13762-013-0229-9.

[19] Brown JN, Peake BM. Determination of colloidally-associated polycyclic aromatic hydrocarbons (PAHs) in fresh water using C18 solid phase extraction disks. Anal Chim Acta, 2003, 486 (2) : 159-169. DOI: 10.1016/S0003-2670 ( 03 ) 00472-0.

[20] Feng C, Xia X, Shen Z. Distribution and sources of polycyclic aromatic hydrocarbons in Wuhan section of the Yangtze River, China. Environ. Monit Assess, 2007, 133(1/2/3) : 447-458. DOI: 10.1007/s10661-006-9599-5.

[21] Amoako J, Ansa-Asare OD, Karikari AY et al. Levels of polycyclic aromatic hydrocarbons (PAHs) in the Densu River Basin of Ghana. Environ Monit Assess, 2011, 174(1/2/3/4) : 471-480. DOI: 10.1007/s10661-010-1471-y.

[22] Zhang L, Dong L, Ren L et al. Concentration and source identification of polycyclic aromatic hydrocarbons and phthalic acid esters in the surface water of the Yangtze River Delta, China. J Environ Sci, 2012, 24(2) : 335-342. DOI: 10.1016/ S1001-0742 (11) 60782-1.

[23] Chen F, Meng FS, Wang YY. The research of polycyclic aromatic hydrocarbons in the river based on the principal component-multivariate linear regression analysis. Environmental Monitoring in China, 2016, 32(4): 49-53. [陈峰, 孟凡生, 王业耀等. 基于主成分分析-多元线性回归的松花江水体中多环芳烃源解析, 中国环境监测, 2016, 32(4): 49-53.]

[24] Wang F, Xia X, Sha Y. Distribution of Phthalic Acid Esters in Wuhan section of the Yangtze River, China. J Hazard Mater, 2008, 154: 317-324. DOI: 10.1016/j.jhazmat.2007.10.028.

[25] Zeng F, Cui K, Xie Z et al. Occurrence of phthalate esters in water and sediment of urban lakes in a subtropical city, Guangzhou, South China. Environ Int, 2008, 34(3) : 372-380. DOI: 10.1016/j.envint.2007.09.002.

[26] Koniecki D, Wang R, Moody RP et al. Phthalates in cosmetic and personal care products: Concentrations and possible dermal exposure. Environ Res, 2011, 111(3) : 329-336. DOI : 10.1016/j.envres.2011.01.013. 